(C) 2018. This manuscript version is made available under the CC-BY-NC-ND 4.0 license http://creativecommons.org/ licenses/by-nc-nd/4.0/

\title{
Accuracy of duplex stainless steel feature generated by electrical discharge machining (EDM)
}

\author{
Ali Alshemary ${ }^{1}$, A Pramanik ${ }^{2 *}$, A. K. Basak ${ }^{3}$, Guy Littlefair ${ }^{4}$ \\ ${ }^{1}$ Reflex Technologies International, Perth, Australia \\ ${ }^{2}$ School of Civil and Mechanical Engineering, Curtin University, Perth, Australia 6102 \\ ${ }^{3}$ Adelaide Microscopy, the University of Adelaide, Adelaide, Australia \\ ${ }^{4}$ Faculty of Design and Creative Technologies, Auckland University of Technology, New Zealand \\ *Contact author: Phone: +61 89266 7981, Fax: +61 89266 2681, Email: alokesh.pramanik@curtin.edu.au
}

\begin{abstract}
The present paper studies different types of errors generated on the feature (cylindrical holes) which was fabricated by the wire EDM of 2205 duplex stainless steel. Different experimental parameters such as, pulse on time, pulse off time and wire tension on the feature as well as the contribution of these parameters on cylindricity error, circularity error and diameter error were explored. Moreover, interactions among the input parameters were also considered. It was found that the total contributions of interactions between different parameters are reasonably high for all the cases which make the modelling process very complex for cylindricity, circularity and diameter errors in term of the range of parameter considered in this investigation. Wire tension has highest contribution on cylindricity error which is lowest at high value wire tension. Pulse on time has minor contribution on the cylindricity error and it increases with the increase of pulse on time. Pulse of time does not have any influence on the cylindricity error. The circularity error was lowest at medium pulse off time and medium wire tension; and those two parameters have almost similar and highest contributions. The pulse on time has around $14 \%$ contribution on circularity error and the medium value of it minimizes the circularity error. The input parameters such has pulse on time, pulse off time and wire tension have around $13 \%, 16 \%$ and $7 \%$ contributions respectively on diameter error which is minimized at medium pulse on time, and low pulse off time and low wire tension.
\end{abstract}

Keywords: Duplex stainless steel; errors; wire EDM.

\section{Introduction}

Stainless steel is a renowned engineering material for number of structural applications. Duplex stainless steel is a special variety of stainless steel which resulted due to continuous development towards obtaining superior properties such as, localised corrosion resistance (J Nomani, Alokesh Pramanik, T Hilditch, \& G Littlefair, 2013), high toughness and good hot workability. Since its introduction in structural applications, the demand 
is ever increasing and supersedes the most popular austenitic stainless steel as a result of low production cost due to the requirement of lower amounts of expensive nickel in it. This is foreseen to continue as recent development specifies evolving applications of such duplex stainless steels in structural design (Baddoo, 2008). Duplex stainless steel usually contains higher and lower level of chromium and sulphur to ensure those properties. Microstructurally, duplex stainless steels have equal amounts of body-centred cubic (BCC) ferrite $(\alpha)$ and face-centred cubic (FCC) austenite $(\gamma)$ phases and thus offers to exploit the benefit of having dual phases simultaneously (Pramanik, Littlefair, \& Basak, 2015). The $\alpha$-ferrite phase has superior resistance to pitting and crevice corrosion whereas $\gamma$-austenite phase promotes strength and toughness. Stainless steels, in general, put an extra challenge during machining operation because of high affinity towards work harden; high toughness and comparatively low thermal conductivity and high fracture toughness (Dolinšek, 2003; Jiang, Paro, Hänninen, Kauppinen, \& Oraskari, 1996; O’Sullivan \& Cotterell, 2002; Paro, Hänninen, \& Kauppinen, 2001). These enhanced material properties upsurge tool/chip interface temperature which causes poor surface finish and frequent breaking of chips. Thus family of stainless steel materials are considered as difficult to machine materials. On top of the above, duplex stainless steel makes the machining scenarios more challenging due to its high strength and to make it worse, duplex stainless steel usually have low carbon content without non-metallic inclusions (Nilsson, 1992; Voronenko, 1997). In addition, duplex stainless steel has a tendency for built-up edge (BUE) formation which reduces machining efficiency due to material adherence to cutting tool and eventually reduce cutting speeds. These result in poor surface finish, low dimensional accuracy and accelerated tool wear (J Nomani, Pramanik, Hilditch, \& Littlefair, 2016, 2017). It is thus apparent that, persistent development in machining technology is foreseen towards further diverse applications of duplex stainless steels. Therefore, a better understanding of the process as a whole might lead to better machining techniques. At present, limited research is available on traditional machining process of these alloys (Junior Nomani, Pramanik, Hilditch, \& Littlefair, 2015). Our previous communication in that area (J. Nomani, A. Pramanik, T. Hilditch, \& G. Littlefair, 2013) identified duplex alloys such as SAF 2507 and SAF 2205 experience higher incidences of built-up edge formation compare to austenite $316 \mathrm{~L}$ which accelerates tool wear and poor surface finish. Paro et al. (Paro et al., 2001) also reported similar results and adhesion wear of machining tool due to BUE formation as predominant tool failure mechanism in their machinability study during drilling of cast duplex stainless steel. Carlborg (Carlborg, 1991) also observed such BUE formation during turning operation and blamed higher ferrite content for prompt BUE formation. The frequent BUE formation is due to the fact that, materials with two phases experience additional fracture along chip-tool rake face whereas single phase materials retain one fracture point only (Williams \& Rollason, 1970).

Though there are complexities in conventional machining of DSS, the non-traditional material removal processes have never been tried for these materials except in (Pramanik, Basak, Dixit, \& Chattopadhyaya, 2018) where only few issues were addressed. Pramanik et al., (Pramanik et al., 2018) investigated WEDM of 
2205 duplex stainless steel where the effects of pulse-on time, wire tension and pulse-off time on surface finish, kerf width and MRR were analysed. However, the other parameters such as, cylindricity, circularity and diameter errors are yet to be investigated as these parameters affect the joining processes and efficiency significantly (Pramanik et al., 2017). Different non-conventional material removal processes are available for a rage of difficult to machine materials (Pramanik, 2014). Among those, electrical discharge machining (EDM) is a commonly used non-conventional method which is highly economical to remove materials by vaporising through locally generated tiny high temperature sparks (Pramanik, Basak, Islam, \& Littlefair, 2015; Pramanik \& Littlefair, 2016). Therefore, this study aims to investigate accuracy of EDM process during machining of DSS at different machining conditions using ANOVA, Taguchi and convention methods. This will benefit researchers and professionals to understand the effectiveness of EDM to remove DSS material.

\section{Methods and materials}

The experiments were conducted using a FANUC ROBOCUT $\propto$-0iD. Grade 2205 Duplex stainless steel disks were in the form of a four inch diameter cylindrical bar. The bar was cut into two disks of $15 \mathrm{~mm}$ thickness each using a hydraulic saw. The top and bottom surfaces of the both bars were machined to have better surface finish, leaving behind two clean and workable $14 \mathrm{~mm}$ thick disks. The disks were then marked with 14 holes each and $4 \mathrm{~mm}$ holes were drilled all the way through. This was aimed at minimising machining time. The top edge was de-burred in an attempt to both mark out top side of disk, as well as avoid any injuries due to sharp edges. Each of those holes were number stamped with the respective trial number. Those holes act as wire-feed positions, so machining can start from the centre of the holes, rather than entering from the outside of the disc and manoeuvring to the centre of the hole. A disk is then fixed to the machines bed. The wire is then fixed in both top and bottom wire-guides and the required pre-set tension is applied by the machine. PC-DMIS coordinate measuring machine (CMM) was used to measure cylindricity, circularity and dimensional errors. The chemical compositions of the workpiece material is given in table 1.

\begin{tabular}{|c|c|c|c|c|c|c|c|c|c|c|c|c|}
\hline $\begin{array}{l}\text { Table } 1 \\
\text { Alloy }\end{array}$ & Cleme & $\begin{array}{l}\text { tal an } \\
\mathbf{M n}\end{array}$ & $\begin{array}{r}\text { ysis } \\
\text { Si }\end{array}$ & $\mathrm{d}_{\mathbf{S}}$ & $\underset{\mathbf{P}}{\operatorname{anica}}$ & $\begin{array}{l}\text { prope } \\
\mathbf{N i}\end{array}$ & ies of & $\begin{array}{c}\text { workp } \\
\text { Mo }\end{array}$ & $\underset{\text { Fe }}{\text { ece mate }}$ & $\begin{array}{l}\text { alUJISor } \\
\text { (MPa) }\end{array}$ & $\begin{array}{l}\text { (MPiedtala } \\
\text { (MPa) }\end{array}$ & $\begin{array}{c}\text { AGrdiness } \\
\text { HV100g }\end{array}$ \\
\hline SAF 2205 & 0.02 & 0.8 & 0.4 & 0.01 & 0.02 & 5.2 & 22.4 & 3.05 & Balance & 777 & 556 & 279 \\
\hline
\end{tabular}

The experimental design was formulated according to Taguchi's orthogonal array method with three-level three-parameter $\mathrm{L}_{27}\left(3^{3}\right)$ as shown in table 2 . The experimental runs $(n=27)$ were conducted, providing interactions between: (i) pulse on and pulse off times, (ii) pulse on time and wire tension and (iii) pulse off time and wire tension of the selected control parameters and levels as summarized in table 3 . The machining 
parameters and the levels of those were selected based on the available information in the literature and capacities of the machine tools available in the laboratory.

Results from this work were analysed using two statistical tools: (i) Pareto ANOVA and (ii) Taguchi's signalto-noise $(S / N)$ ratio analysis. Pareto ANOVA identified the degree to which each control parameter affects the cylindricity, circularity and dimension errors of the machined workpiece based on Pareto principle that only $20 \%$ of the total machining configurations would be required to generate $80 \%$ of the benefits of completing every test configuration (Haughey, 2017). Pareto ANOVA is a simplified form of ANOVA which neither requires an ANOVA table nor the use of $F$-tests. Therefore, detailed knowledge of the ANOVA method is not required. An elaborate discussion on Pareto ANOVA method can be found in open literature (Park, 1996).

Table 2 Machining parameters and their respective levels according to Taguchi's method.

\begin{tabular}{ccccccc}
\hline Input parameter & & Symbol & & Level 0 & Level 1 & Level 2 \\
\cline { 1 - 3 } \cline { 5 - 7 } Pulse-on time $(\mu \mathrm{s})$ & & $\mathrm{A}$ & & 4 & 6 & 8 \\
Pulse-off time $(\mu \mathrm{s})$ & & $\mathrm{B}$ & & 22 & 44 & 66 \\
Wire Tension $(\mathrm{gf})$ & & $\mathrm{C}$ & & 1000 & 1400 & 1800 \\
\hline
\end{tabular}

In the present study, authors use Taguchi's statistical method (Taguchi, 1987) to establish optimum cutting parameters allowing the machining parameters to be vigorously established on machining outputs (Pramanik et al., 2016). Taguchi method applies $S / N$ ratio to enhance the outcome of a manufacturing method according to equation 1:

$S / N=-10 \log \frac{1}{n}\left(\sum_{i=1}^{n} y_{i}^{2}\right)$

Here, observation number is $n$ and observed data is $y$. The above equation is appropriate for quality characteristics in which the saying "the bigger the better" is true. Equation 1 also implies that, greater the magnitude of $S / N$ ratio, better the result will be as it produces the top quality with smallest variance.

Table 3 Experiments details

\begin{tabular}{cccc}
\hline Expt. no. & Pulse-on time $(\mu \mathrm{s})$ & Pulse-off time $(\mu \mathrm{s})$ & Wire Tension $(\mathrm{gf})$ \\
\hline 1 & 4 & 22 & 1000 \\
2 & 4 & 22 & 1400 \\
3 & 4 & 22 & 1800 \\
4 & 4 & 44 & 1000 \\
5 & 4 & 44 & 1400 \\
6 & 4 & 44 & 1800 \\
7 & 4 & 66 & 1000 \\
8 & 4 & 66 & 1400 \\
9 & 4 & 66 & 1800
\end{tabular}




\begin{tabular}{llll}
10 & 6 & 22 & 1000 \\
11 & 6 & 22 & 1400 \\
12 & 6 & 22 & 1800 \\
13 & 6 & 44 & 1000 \\
14 & 6 & 44 & 1400 \\
15 & 6 & 44 & 1800 \\
16 & 6 & 66 & 1000 \\
17 & 6 & 66 & 1400 \\
18 & 6 & 66 & 1800 \\
19 & 8 & 22 & 1000 \\
20 & 8 & 22 & 1400 \\
21 & 8 & 22 & 1800 \\
22 & 8 & 44 & 1000 \\
23 & 8 & 44 & 1400 \\
24 & 8 & 44 & 1800 \\
25 & 8 & 66 & 1000 \\
26 & 8 & 66 & 1400 \\
27 & 8 & 66 & 1800 \\
\hline
\end{tabular}

3. Result

Dimensional accuracy of machined features that are 'holes' in the workpiece in present study, are represented by cylindricity, circularity and diameter errors as the most important parameters. These terms are explained as follows: the dissimilarity between measured and designed diameters is diameter error. Undercutting of the hole induces negative error. The diameter error of a machined hole plays most important role during tight fit of the components. Another vital quality characteristic is circularity also well-known as roundness. This is measured by the distance between rims of two concentric circles in which each circular component of the surface should be located (ASME, 2009). The cylindricity represents the circularity along the depth of the hole. Too much circularity error generates undesirable vibration and heat in rotating parts (Pramanik et al., 2016). Thus, these important parameters are engaged to understand the appropriateness of EDM technique for fabricating holes in duplex stainless steel.

\subsection{Cylindricity}

Cylindricity is a measure of how much a cylindrical surface varies from an ideal cylinder that is perfectly round, perfectly straight and has no taper (Engineering Essentials, 2015). The Pareto ANOVA for cylindricity error shown in table 4 signifies that wire tension $(\mathrm{C})$ affected $(\mathrm{P} \cong 21 \%)$ cylindricity most significantly. The second most contributing parameter is interaction between pulse-off time and wire tension $(\mathrm{B} \times \mathrm{C})$ which contributes $\mathrm{P} \cong 19 \%$, this is followed by interactions between pulse-on time and pulse-off time $(\mathrm{A} \times \mathrm{B})$, and pulse-on time and wire tension $(\mathrm{A} \times \mathrm{C})$ with contributions, $\mathrm{P} \cong 18$ and $16 \%$ respectively. In addition to wire tension, the other only single parameter pulse-on time (A) contributes only $\mathrm{P} \cong 10 \%$. The rest of the 
contribution comes from the interactions of parameters. The total impact of main effects or effect of individual parameter is around $31 \%$ and on the other hand, the total influence of interaction effects is $70 \%$. Therefore, it is extremely difficult to optimise the cylindricity errors in this case by selecting input parameters.

Table 4 Investigation of cylindricity error by Pareto ANOVA

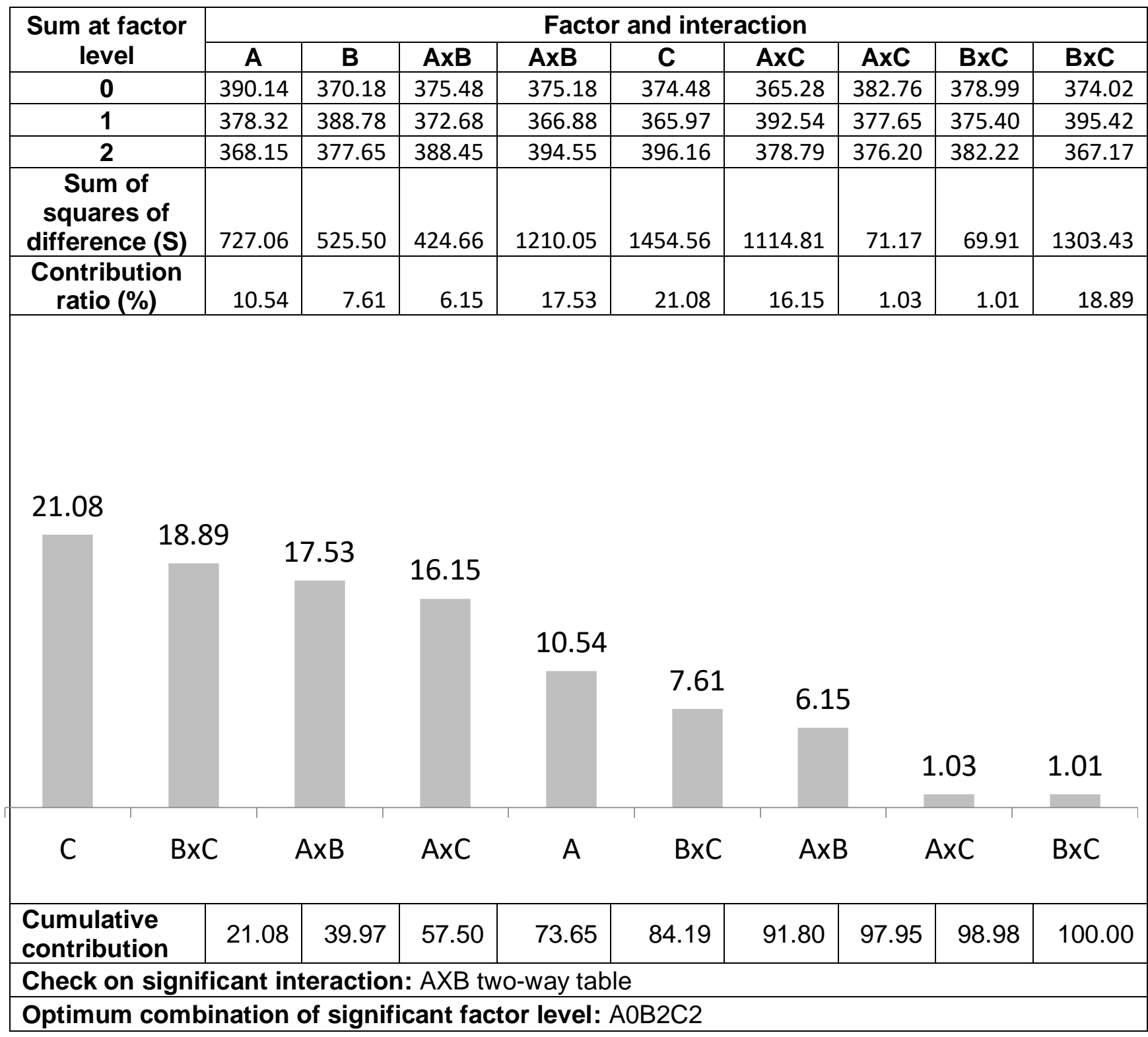

Response graph in figure 1 represents the outcomes of Pareto ANOVA, as shown in table 5, and confirms that high level of wire tension (C2) is the best variable for minimum cylindricity error. Based on the two-way table (details in appendix) of $\mathrm{B} \times \mathrm{C}$, optimal arrangement of $\mathrm{B}$ and $\mathrm{C}$ to attain lowermost cylindricity error is found as B2C2. Consequently, the paramount arrangement of input parameters for minimal cylindricity error is noted as A0B2C2. Though the individual contributions of pulse-on time (A) and pulse-off time (B) are negligible, those give lower cylindricity at maximum and medium values, respectively. The variations in cylindricity error for all the variables are given in figure 2. The optimal outputs are attained at low pulse-on time. Medium pulse-off time gives lower cylindricity error compare to that of high and low values of pulse-off time. The 
cylindricity error increases with the rise of wire tension but it is minimized when the wire tension increases further. These observations are quite similar to those attained from response tables and Pareto ANOVA.

Table 5 Response table for mean $\mathrm{S} / \mathrm{N}$ ratios for important interactions and cylindricity error

\begin{tabular}{|c|c|c|c|c|c|}
\hline Input parameters & Symbol & Level 0 & Level 1 & Level 2 & max-min \\
\hline Pulse-on time $(\mu \mathrm{s})$ & $\mathrm{A}$ & 43.35 & 42.04 & 40.91 & 2.44 \\
\hline Pulse-off time $(\mu \mathrm{s})$ & $\mathrm{B}$ & 41.13 & 43.20 & 41.96 & 2.07 \\
\hline Wire Tension $(\mathrm{gf})$ & $\mathrm{C}$ & 41.61 & 40.66 & 44.02 & 3.36 \\
\hline Interaction & $\mathrm{BXC}$ & 42.11 & 41.71 & 42.47 & 0.76 \\
\hline
\end{tabular}

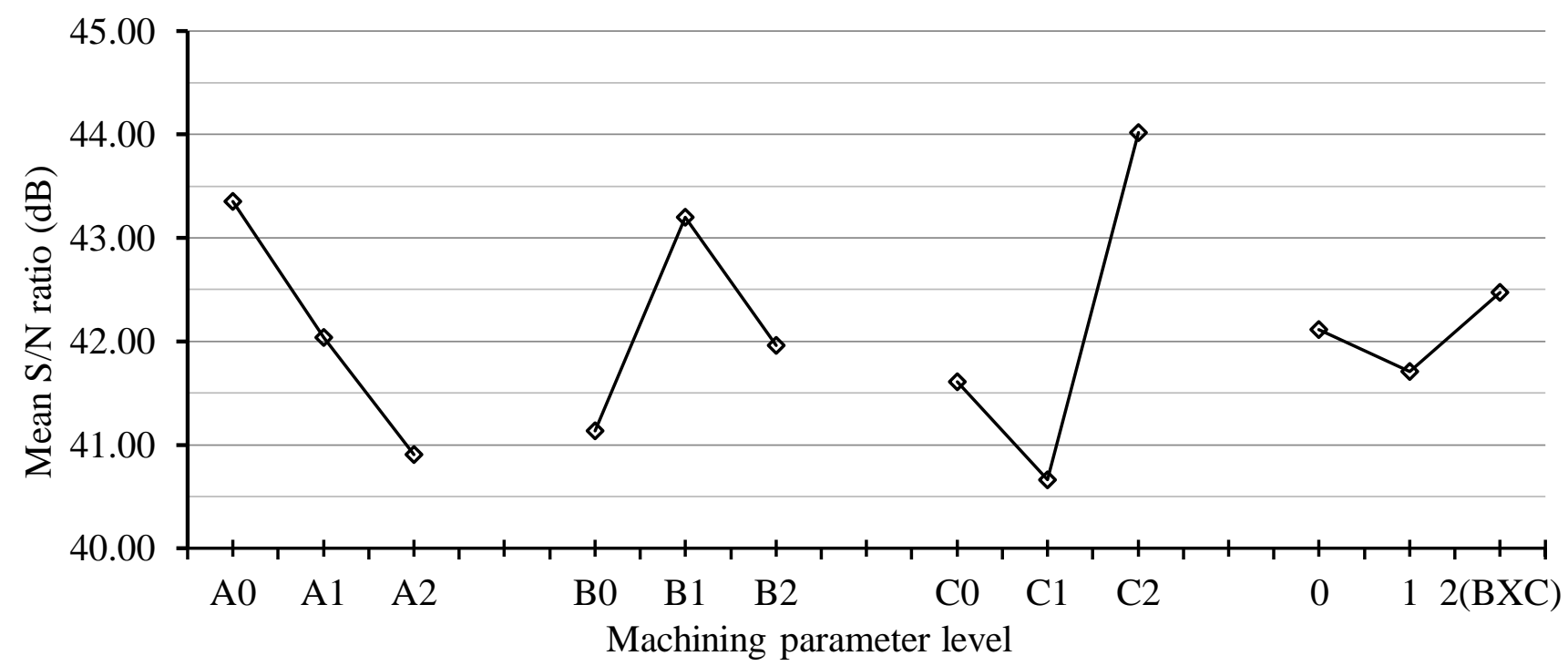

Fig 1. Response graph of $\mathrm{S} / \mathrm{N}$ ratios for cylindricity error

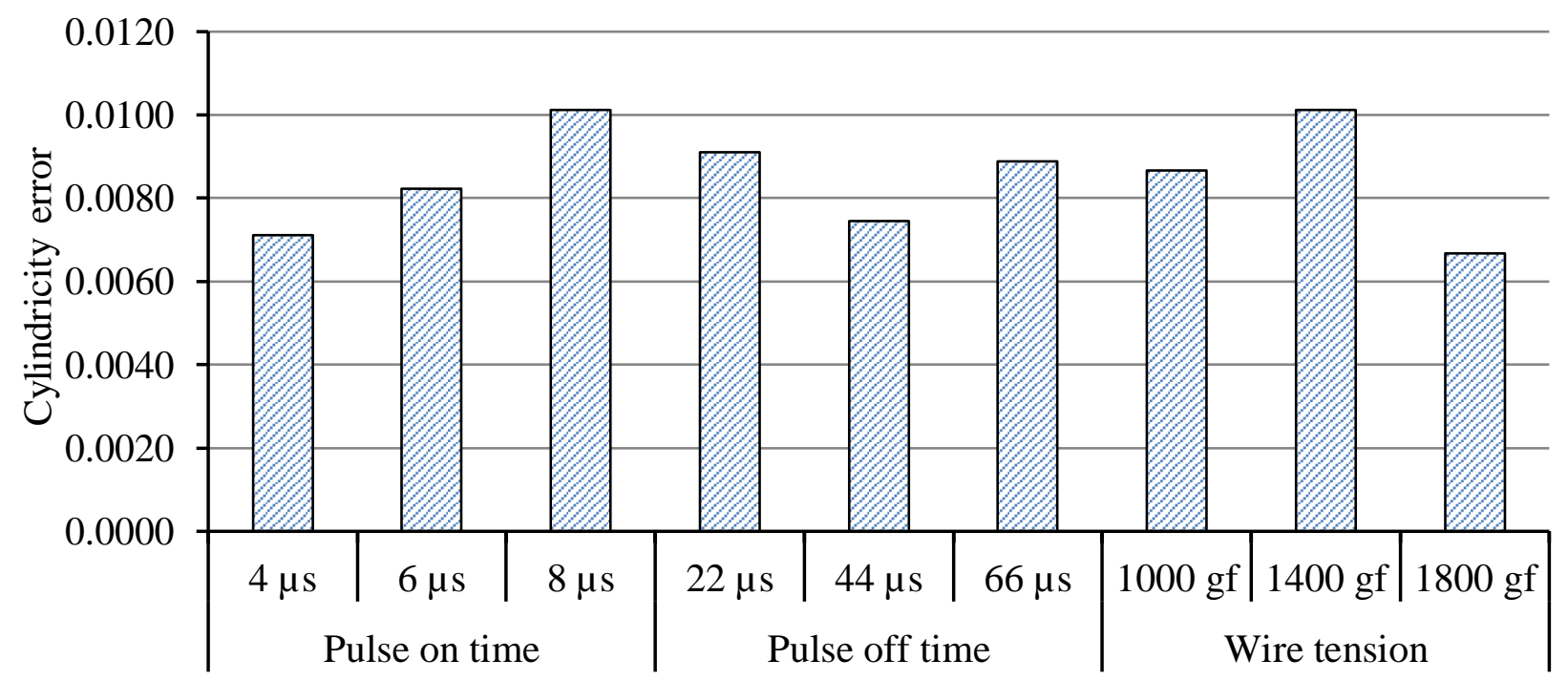

Fig. 2 Average cylindricity errors for all the variables

3.2 Circularity error 
Pareto ANOVA analysis of circularity error shown in table 6 points out that the individual contributions of pulse-off time (B) and wire tension (C) are almost similar and most significant with $\mathrm{P} \cong 19 \%$. The third most important aspect is the interaction between pulse-on time and pulse-off time $(\mathrm{A} \times \mathrm{B})$ with $\mathrm{P} \cong 15 \%$, followed by the pulse-on time $(\mathrm{A})(\mathrm{P} \cong 14 \%)$ and the rest are the diverse interactions between different parameters. The entire influence of individual parameters is about $53 \%$, but the contribution of interactions is $47 \%$ in this case. Therefore, it is very problematic to find optimal circularity error by choosing individual input variables.

Response data presented in Fig. 3 endorses the outcomes of the Pareto ANOVA shown in table 7. The low level of pulse-on time (A0) is the best arrangement as presented in table 7. Because of the significant interaction between $\mathrm{A}$ and $\mathrm{B}, \mathrm{A} \times \mathrm{B}$ two-way table is used to find the combination of these two parameters (see Appendix). The two-way table shows that the optimal arrangement of A and B to obtain the least circularity error is A1B1. Therefore, the optimal arrangement of input parameters is A1B1C1 for lowest circularity error. This is medium levels of pulse-on time ( $6 \mu \mathrm{s})$, pulse-off time (44 $\mu \mathrm{s})$ and wire tension (1400 gf).

The variation of the circularity error for three input parameters is presented in figure 4. The circularity error reduces with the increase of all three parameters and continue to rise with the further increase of these parameters. Therefore, the errors were minimized at medium values of input parameters. These outcomes are very similar to those attained from response tables as well as Pareto ANOVA.

Table 6 Pareto ANOVA for circularity error

\begin{tabular}{|c|c|c|c|c|c|c|c|c|c|}
\hline \multirow{2}{*}{ Sum at factor level } & \multicolumn{9}{|c|}{ Factor and interaction } \\
\cline { 2 - 11 } & A & B & AxB & AxB & C & AxC & AxC & BxC & BxC \\
\hline $\mathbf{0}$ & 349.16 & 343.91 & 360.44 & 372.52 & 349.54 & 351.61 & 353.10 & 365.76 & 356.03 \\
\hline $\mathbf{1}$ & 371.42 & 369.21 & 357.69 & 351.69 & 374.19 & 367.39 & 356.83 & 363.79 & 368.29 \\
\hline $\mathbf{2}$ & 356.63 & 364.08 & 359.08 & 353.00 & 353.48 & 358.21 & 367.28 & 347.66 & 352.89 \\
\hline $\begin{array}{c}\text { Sum of squares of } \\
\text { difference (S) }\end{array}$ & 770.43 & 1073.20 & 11.33 & 817.10 & 1051.40 & 377.00 & 324.06 & 591.57 & 397.06 \\
\hline $\begin{array}{c}\text { Contribution ratio } \\
\text { (\%) }\end{array}$ & 14.23 & 19.83 & 0.21 & 15.09 & 19.42 & 6.96 & 5.99 & 10.93 & 7.34 \\
\hline
\end{tabular}

\section{$19.83 \quad 19.42$}

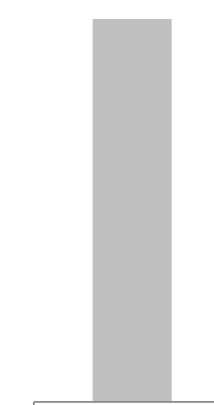

15.09 14.23

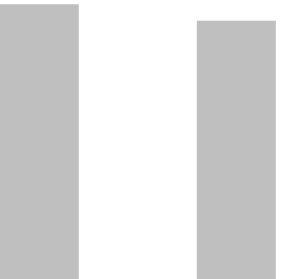

10.93

B

$A \times B$

A

BxC

BxC

7.34

6.93

5.99

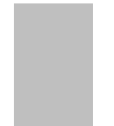

0.21 
Check on significant interaction: AXB two-way table

Optimum combination of significant factor level : A1B1C1

Table 7 Response table for mean $\mathrm{S} / \mathrm{N}$ ratios for circularity error and significant interactions

\begin{tabular}{|c|c|c|c|c|c|}
\hline Input parameters & Symbol & Level 0 & Level 1 & Level 2 & max-min \\
\hline Pulse-on time $(\mu \mathrm{s})$ & $\mathrm{A}$ & 38.80 & 41.27 & 39.63 & 2.47 \\
\hline Pulse-off time $(\mu \mathrm{s})$ & $\mathrm{B}$ & 38.21 & 41.02 & 40.45 & 2.81 \\
\hline Wire Tension $(\mathrm{gf})$ & $\mathrm{C}$ & 38.84 & 41.58 & 39.28 & 2.74 \\
\hline Interaction & AXB & 41.39 & 39.08 & 39.22 & 2.31 \\
\hline
\end{tabular}

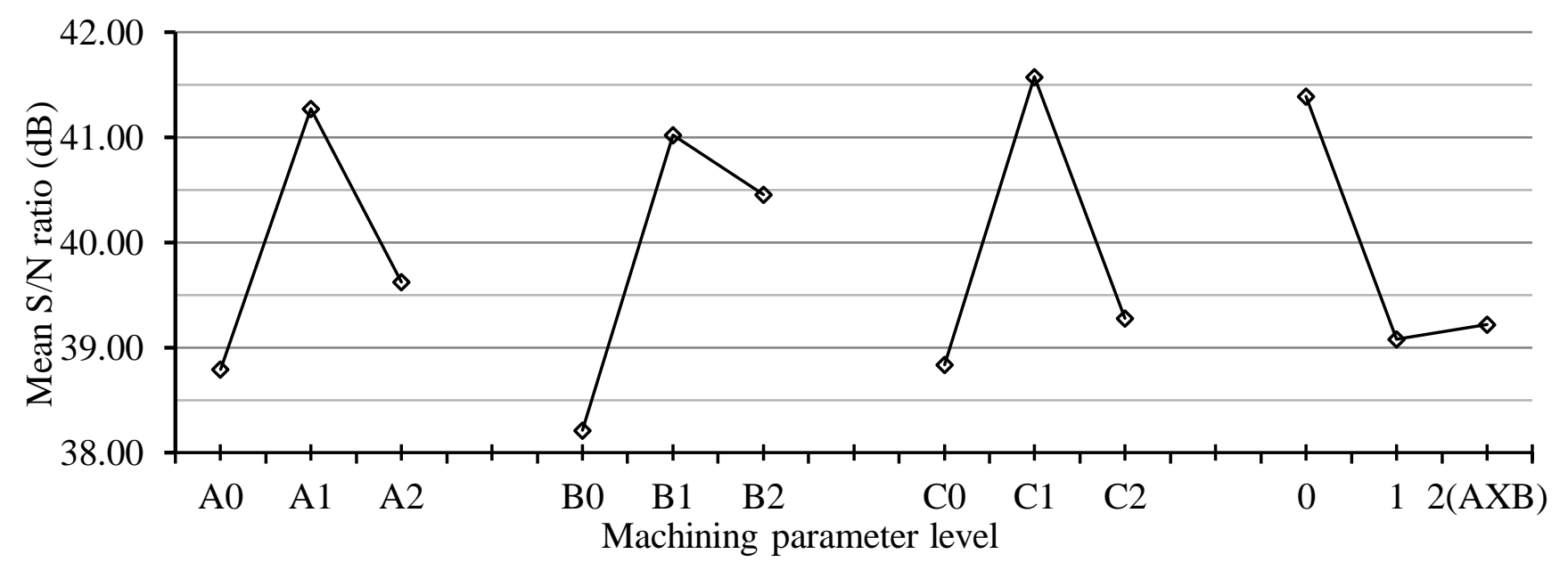

Fig. 3 Response data of $\mathrm{S} / \mathrm{N}$ ratios for circularity

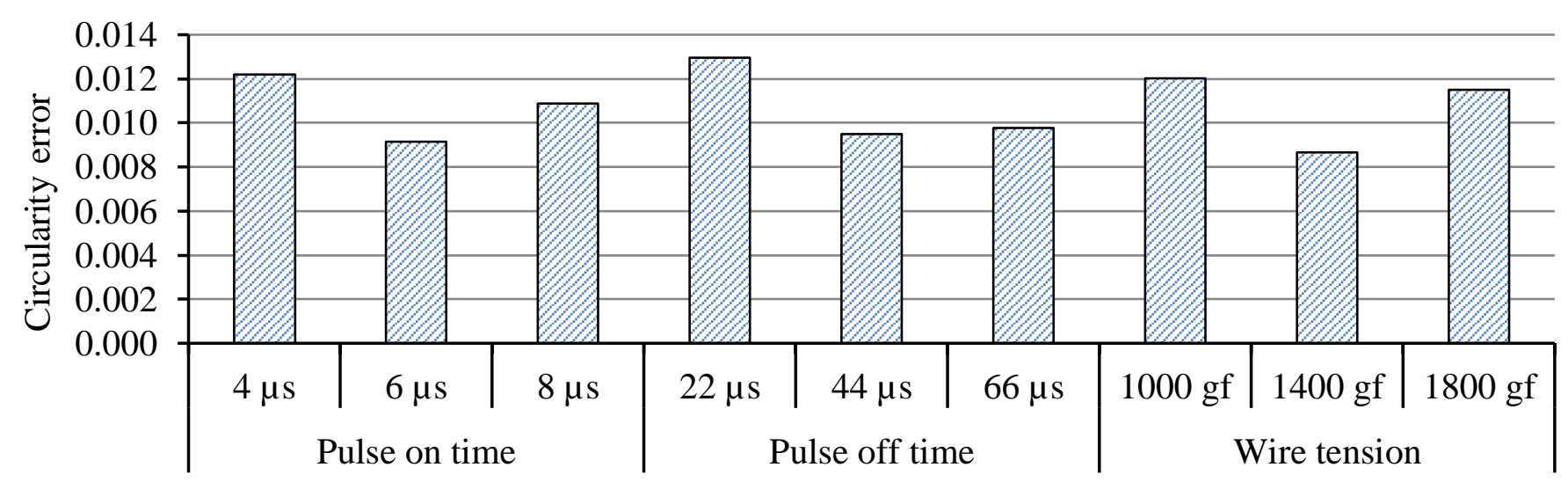

Fig. 4 Average circularity errors

\subsection{Diameter error}

Pareto AVOVA examination of diameter error presented in table 8 clearly exposes that the most significant contributor is the first interaction between pulse-off time and wire tension $(\mathrm{B} \times \mathrm{C})(\mathrm{P} \cong 19 \%)$, and second interaction amongst pulse-off time and wire tension $(\mathrm{B} \times \mathrm{C})(\mathrm{P} \cong 16 \%)$ which is very similar to that of pulseoff time (B). The individual influence of pulse-on time (A) and wire tension (C) contribute around $13 \%$ and 
$7 \%$ correspondingly. It is worth to note that, entire effect of individual parameter is around $36 \%$, but the entire influence of the interactions around $64 \%$, thus it is also complex to optimize the diameter error by selecting input variables.

The response data for diameter error is shown in table 9 and figure 5, with the gradients of response graphs, representing the power to impact as established by ANOVA study in table 9. The medium pulse-on time (A1) was in the top to attain a lowest diameter error as presented in figure 5. Subsequently the interaction of $A \times B$ is substantial. Thus, two-way table of A and B has been used to find optimal levels of A and B (see Appendix). The optimum combination of factors A and B in order to achieve lowest diameter error is determined as A1B0. As a result, the best arrangement of input parameters to minimise circularity error is A1B0C0. This is medium level of pulse-on time $(6 \mu \mathrm{s})$, lowest level of pulse-off time $(22 \mu \mathrm{s})$, and low level of wire tension (1000 gf).

Table 8 Pareto ANOVA analysis for diameter error

\begin{tabular}{|c|c|c|c|c|c|c|c|c|c|}
\hline \multirow{2}{*}{$\begin{array}{c}\text { Sum at factor } \\
\text { level }\end{array}$} & \multicolumn{9}{|c|}{ Factor and interaction } \\
\cline { 2 - 11 } & $\mathbf{A}$ & $\mathbf{B}$ & $\mathbf{A x B}$ & $\mathbf{A x B}$ & $\mathbf{C}$ & $\mathbf{A x C}$ & AxC & BxC & BxC \\
\hline $\mathbf{0}$ & 345.22 & 370.61 & 336.36 & 359.05 & 365.22 & 355.30 & 352.42 & 338.50 & 336.50 \\
\hline $\mathbf{1}$ & 369.19 & 343.93 & 368.75 & 344.41 & 350.02 & 350.51 & 353.08 & 365.74 & 363.74 \\
\hline $\mathbf{2}$ & 349.20 & 349.07 & 358.50 & 360.15 & 348.37 & 357.80 & 358.10 & 359.36 & 363.37 \\
\hline $\begin{array}{c}\text { Sum of squares } \\
\text { of difference (S) }\end{array}$ & 989.86 & 1202.78 & 1644.53 & 463.20 & 517.41 & 82.21 & 57.94 & 1217.57 & 1463.85 \\
\hline $\begin{array}{c}\text { Contribution ratio } \\
\text { (\%) }\end{array}$ & 12.96 & 15.74 & 21.53 & 6.06 & 6.77 & 1.08 & 0.76 & 15.94 & 19.16 \\
\hline
\end{tabular}

\subsection{3} 19.16

\begin{tabular}{|c|c|c|c|c|c|c|c|c|c|c|}
\hline \multirow{4}{*}{ 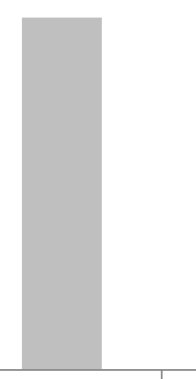 } & \multirow{4}{*}{19.16} & \multirow{4}{*}{15.9} & \multirow{2}{*}{\multicolumn{2}{|c|}{15.74}} & \multirow[b]{2}{*}{12.96} & \multirow[b]{3}{*}{6.77} & \multirow{3}{*}{\multicolumn{2}{|c|}{6.06}} & \multirow[b]{4}{*}{1.08} & \multirow[b]{4}{*}{0.76} \\
\hline & & & & & & & & & & \\
\hline & & & & & & & & & & \\
\hline & & & & & & & & & & \\
\hline$A \times B$ & $\mathrm{BxC}$ & & & B & A & C & $A x$ & & $A \times C$ & $\mathrm{AxC}$ \\
\hline $\begin{array}{l}\text { Cumulative } \\
\text { contribution }\end{array}$ & & 21.53 & 40.69 & 56.63 & 72.37 & 85.33 & 92.10 & 98.16 & 99.24 & 100.00 \\
\hline
\end{tabular}

Table 9 Response table for mean $\mathrm{S} / \mathrm{N}$ ratios for circularity error and significant interactions

\begin{tabular}{|c|c|c|c|c|c|}
\hline Input parameters & Symbol & Level 0 & Level 1 & Level 2 & max-min \\
\hline Pulse-on time $(\mu \mathrm{s})$ & $\mathrm{A}$ & 38.36 & 41.02 & 38.80 & 2.66 \\
\hline Pulse-off time $(\mu \mathrm{s})$ & $\mathrm{B}$ & 41.18 & 38.21 & 38.79 & 2.97 \\
\hline Wire Tension $(\mathrm{gf})$ & $\mathrm{C}$ & 40.58 & 38.89 & 38.71 & 1.87 \\
\hline
\end{tabular}




\begin{tabular}{|c|c|c|c|c|c|}
\hline Interaction & AXB & 39.89 & 38.27 & 40.02 & 1.75 \\
\hline
\end{tabular}

The average deviation of diameter error through conventional method presents that the medium pulse-on time offers smallest errors as presented in Fig. 6. The minimum pulse-off time gives lowest diameter error though the diameter error increases and then reduces with further increase of pulse-off time. The diameter error reduces with the reduction of wire tension, and minimum at lowermost wire tension (1000 gf).

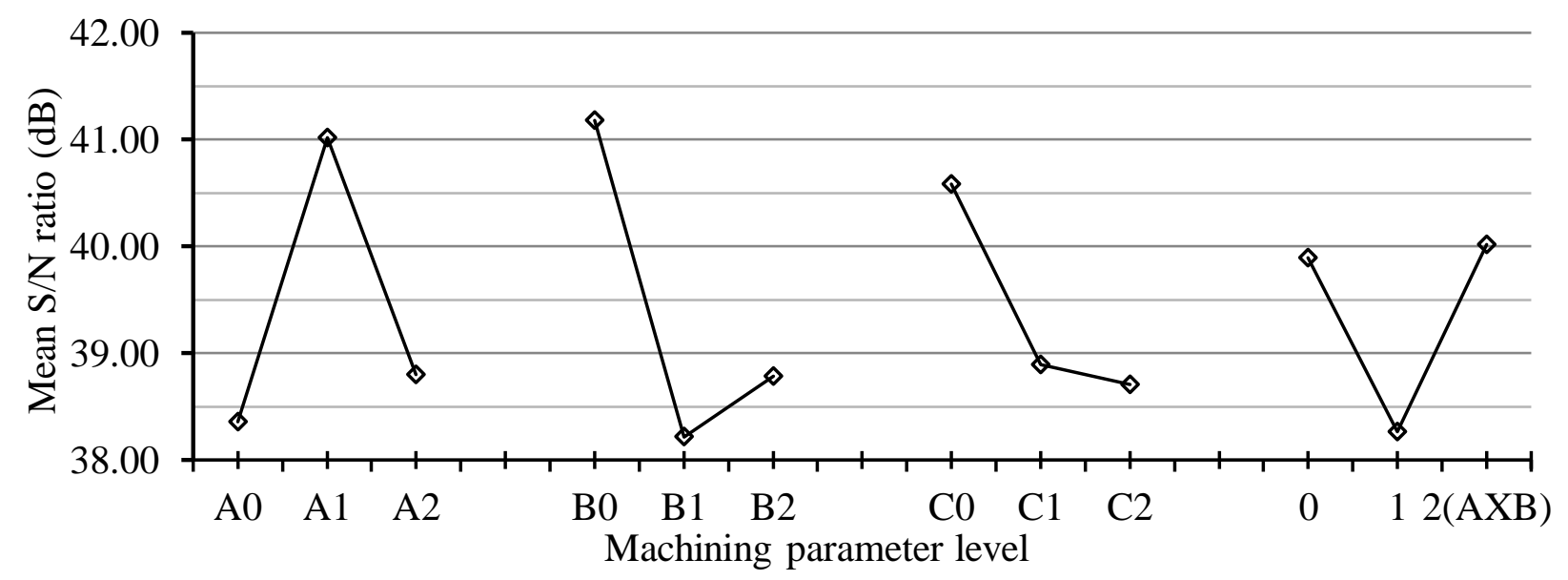

Fig. 5 Response graph of $\mathrm{S} / \mathrm{N}$ ratios for diameter error

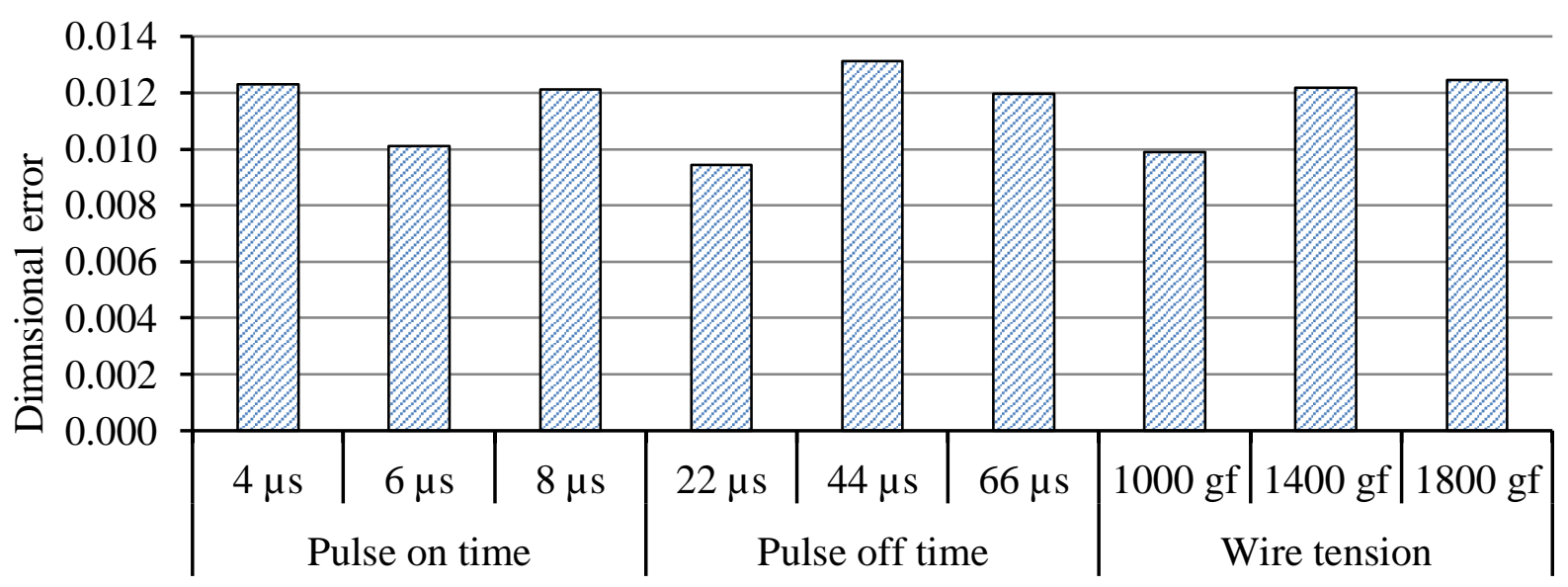

Fig. 6 Average diameter errors for three parameters

\section{Discussion}

During EDM process, material is removed by discrete electric sparks that occur in cycles. Every cycle has an off-time and on-time in microseconds range. As material is being melted and vaporized during pulse on-time or pulse duration, the length and frequency of these pulses are important. Material removal rate is directly proportionate to the induced energy of on-time which further depends on peak current and on-time length (Kansal, Singh, \& Kumar, 2005; Lee \& Tai, 2003). Though more material will be melted away with longer pulse duration, resulting crater will also be broader and deeper. These will result rougher surface finish compared to that produced by shorter pulse duration. Moreover, extended pulse duration also allows more heat to transfer into the work piece and broaden heat affected zone. However, excessive pulse duration is counter-productive as material removal rate will be reduced. A longer period may also put the electrode into a no-wear state and the electrode grows from plating build-up. Therefore, pulse off-time which is also 
known as pulse interval is required for efficient material removal. The smaller the interval, the quicker will be the machining process. However, in case of too short duration, flow of dielectric fluid may not take away the removed material and will not be deionized to stabilize for next spark (Kumar \& Choudhury, 2007; Singh \& Singh, 2012). Such unstable conditions in the process may induce inconsistent cycles and retracts the advancing servo. This slows down material removal more than that for longer and stable off-times. Therefore, pulse break must be longer compared to deionization time to avert continual sparking at one point (Fuller, 1989). Modern power supplies in EDM equipment allow self-determining setting of pulse on-times and off-times with a typical range of 2 to $1000 \mu \mathrm{s}$. Though in ideal situations, each pulse should generate a spark, practically lots of pulses fail if period and intermission are not appropriately synchronized, resulting reduced machining efficiency (Singh \& Singh, 2013). The surface roughness on machined workpiece is generated as uneven fusing structure, globules of debris, shallow craters, pockmarks, cracks and voids. These defects become further prominent with the increase of pulse-on period and pulse current (Pramanik \& Basak, 2016; Alokesh Pramanik et al., 2015). The tension in wire influences the distortion and rigidity of wire electrode. The wire electrode is less rigid and doesn't distort at lesser tension. Therefore a lengthier and broader area of wire takes part to remove material. On the other hand, wire becomes very stiff and its diameter will be reduced at higher tension, resulting shorter and narrower area to take part in material removal process and thus reduce material removal rate at higher tension. In addition, too much tension in wire electrode may deteriorate its physical properties and reduce the capacity to produce expected spark. Conversely, too flexible wire may be unable to retain required spark gap alongside its length for effective removal of material (Alokesh Pramanik et al., 2015; Pramanik et al., 2016; Pramanik \& Littlefair, 2016).

The cylindricity error, a three-dimensional parameter, of a hole generated from wire EDM process depends on the straightness of the wire electrode which is achieved at high wire tension. Therefore, the contribution of the wire tension is maximum on the cylindricity error and minimum error occurs at higher wire tension. For the considered range, the decrease of pulse-on time reduces the cylindricity error by minimizing the material melting uniformly along the length of the wire electrode. The pulse off time does not have any noticeable contribution on the cylindricity error though the medium value of this parameter minimises the cylindricity error. The circularity error which is a two dimensional parameter, depends on the ability of the wire electrode to follow circular arc. The characteristics of wire EDM processes associated to the high and low values of the input parameters is already mentioned. It seems that the medium values of pulse on time, pulse off time and wire tension are most suitable to generate a better circle in this case. The diameter error is induced due to undercut or overcut during the machining process. The data shows that the diameter error in this case is due to over cut which is due to larger kerf width compare to the diameter of wire electrode (Pramanik \& Basak, 2016). Pulse on time is a deciding factor of induced energy and the pulse off time facilitates the material removal process. Therefore, the interaction of these two parameters contributes mostly on diameter error.

\section{Conclusion}

Wire EDM of duplex 2205 stainless steel was investigated by generating cylindrical holes at different machining conditions. The following conclusions can be drawn from this study.

(a) Interactions between parameters as well as individual parameters affect the cylindricity, circularity and diameter errors significantly of holes machined in duplex 22125 stainless steel. The contribution of all interactions is 
significantly high compare to that of individual parameters for all the cases which indicates that the modelling of the cylindricity, circularity and diameter errors in terms of pulse on time, pulse of time and wire tension for the range of parameter considered in this investigation is very complex.

(b) The tension in wire influences the distortion and rigidity of wire electrode where the lesser tension induces higher flexibility. Therefore, wire tension is the most contributing parameter for the cylindricity error which is minimized at higher wire tension. Pulse on time has minor contribution on the cylindricity error which increases with the increase of pulse on time. Pulse of time does not have any influence on the cylindricity error.

(c) The circularity errors comes into effect due to the non-uniform undercut and overcut which are controlled by input parameters. Pulse off time and wire tension have almost similar and highest contribution on the circularity error which is minimized at medium pulse off time and wire tension. The contribution of pulse on time in this case is around $14 \%$, the medium value of which also minimizes the circularity error.

(d) The diameter errors occur due to undercut or overcut. The contributions of the pulse on time, pulse off time and wire tension on diameter errors are around 13,16 and 7\% respectively. The medium pulse on time, and low pulse off time and low wire tension minimize the diameter error.

Appendix:

BC two-way table for cylindricity

\begin{tabular}{|l|l|l|l|l|l|l|l|l|l|l|l|l|l|l|}
\hline & & & B0 & & & & B1 & & & & & B2 & \\
\hline C0 & 38.416 & 41.938 & 40.000 & $\mathbf{1 2 0 . 3 5}$ & 40.915 & 44.437 & 37.721 & $\mathbf{1 2 3 . 0 7}$ & 46.021 & 41.938 & 43.10 & $\mathbf{1 3 1 . 0 6}$ \\
\hline C1 & 41.938 & 44.437 & 37.077 & $\mathbf{1 2 3 . 4 5}$ & 41.938 & 40.915 & 46.021 & $\underline{\mathbf{1 2 8 . 8 7}}$ & 46.021 & 37.721 & 33.98 & $\mathbf{1 1 7 . 7 2}$ \\
\hline C2 & 44.437 & 40.000 & 41.938 & $\mathbf{1 2 6 . 3 7}$ & 44.437 & 37.721 & 40.915 & $\mathbf{1 2 3 . 0 7}$ & 46.021 & 40.915 & 41.94 & $\mathbf{1 2 8 . 8 7}$ \\
\hline
\end{tabular}

AB two-way table for circularity error

\begin{tabular}{|l|l|l|l|l|l|l|l|l|l|l|l|l|l|l|}
\hline & & & A0 & & & & A1 & & & & & A2 & \\
\hline B0 & 35.739 & 44.437 & 36.100 & $\mathbf{1 1 6 . 2 7 6}$ & 39.439 & 41.938 & 34.895 & $\mathbf{1 1 6 . 2 7 2}$ & 37.501 & 37.947 & 35.92 & $\mathbf{1 1 1 . 3 7}$ \\
\hline B1 & 37.077 & 37.077 & 42.694 & $\mathbf{1 1 6 . 8 4 9}$ & 45.460 & 42.694 & 43.522 & $\mathbf{1 3 1 . 6 7 6}$ & 36.873 & 43.522 & 40.29 & $\mathbf{1 2 0 . 6 9}$ \\
\hline B2 & 35.918 & 41.938 & 38.178 & $\mathbf{1 1 6 . 0 3 4}$ & 41.243 & 41.938 & 40.294 & $\mathbf{1 2 3 . 4 7 6}$ & 40.294 & 42.694 & 41.58 & $\mathbf{1 2 4 . 5 7}$ \\
\hline
\end{tabular}

AB two-way table for diameter error

\begin{tabular}{|l|l|c|c|c|c|c|c|c|c|c|c|c|}
\hline & \multicolumn{2}{|c|}{ A0 } & & & \multicolumn{3}{c|}{ A1 } & & \multicolumn{3}{|c|}{ A2 } & \\
\hline B0 & 37.501 & 39.715 & 38.661 & $\mathbf{1 1 5 . 8 7}$ & 40.294 & 46.021 & 48.715 & $\mathbf{1 3 5 . 0 3}$ & 37.947 & 43.098 & 38.66 & $\mathbf{1 1 9 . 7 1}$ \\
\hline B1 & 39.439 & 35.739 & 37.721 & $\mathbf{1 1 2 . 8 9}$ & 47.264 & 38.416 & 36.673 & $\mathbf{1 2 2 . 3 5}$ & 38.178 & 34.579 & 35.92 & $\mathbf{1 0 8 . 6 7}$ \\
\hline B2 & 41.243 & 36.287 & 38.913 & $\mathbf{1 1 6 . 4 4}$ & 38.913 & 37.501 & 35.391 & $\mathbf{1 1 1 . 8 0}$ & 44.437 & 38.661 & 37.72 & $\mathbf{1 2 0 . 8 2}$ \\
\hline
\end{tabular}

\section{Acknowledgement}

The authors' sincerely acknowledges the helps of Dr M. N. Islam on statistical methods. 


\section{References}

ASME. (2009). Dimensioning and Tolerancing ASME. New York: ASME.

Baddoo, N. (2008). Stainless steel in construction: A review of research, applications, challenges and opportunities. Journal of Constructional Steel Research, 64(11), 1199-1206.

Carlborg, C. (1991). Machinability of duplex stainless steel. Proceedings of duplex stainless steel, 1(1), 683-696.

Dolinšek, S. (2003). Work-hardening in the drilling of austenitic stainless steels. Journal of Materials Processing Technology, 133(1), 63-70.

Fuller, J. E. (1989). Electrical discharge machining. ASM Handbook., 16, 557-564.

Haughey, D. (2017). Pareto Analysis Step by Step. Retrieved from http://www.projectsmart.co.uk/pareto-analysisstep-by-step.html

Jiang, L., Paro, J., Hänninen, H., Kauppinen, V., \& Oraskari, R. (1996). Comparison of grindability of HIPped austenitic $316 \mathrm{~L}$, duplex 2205 and super duplex 2507 and as-cast 304 stainless steels using alumina wheels. Journal of Materials Processing Technology, 62(1-3), 1-9.

Kansal, H., Singh, S., \& Kumar, P. (2005). Parametric optimization of powder mixed electrical discharge machining by response surface methodology. Journal of Materials Processing Technology, 169(3), 427-436.

Kumar, S., \& Choudhury, S. (2007). Prediction of wear and surface roughness in electro-discharge diamond grinding. Journal of Materials Processing Technology, 191(1), 206-209.

Lee, H.-T., \& Tai, T. Y. (2003). Relationship between EDM parameters and surface crack formation. Journal of Materials Processing Technology, 142(3), 676-683.

Nilsson, J.-O. (1992). Super duplex stainless steels. Materials science and technology, 8(8), 685-700.

Nomani, J., Pramanik, A., Hilditch, T., \& Littlefair, G. (2013). Machinability study of first generation duplex (2205), second generation duplex (2507) and austenite stainless steel during drilling process. Wear, 304(1), 20-28.

Nomani, J., Pramanik, A., Hilditch, T., \& Littlefair, G. (2013). Machinability study of first generation duplex (2205), second generation duplex (2507) and austenite stainless steel during drilling process. Wear, 304(1-2), 20-28. doi:http://dx.doi.org/10.1016/j.wear.2013.04.008

Nomani, J., Pramanik, A., Hilditch, T., \& Littlefair, G. (2015). Chip formation mechanism and machinability of wrought duplex stainless steel alloys. The International Journal of Advanced Manufacturing Technology, 80(5-8), 11271135.

Nomani, J., Pramanik, A., Hilditch, T., \& Littlefair, G. (2016). Investigation on the behavior of austenite and ferrite phases at stagnation region in the turning of duplex stainless steel alloys. Metallurgical and Materials Transactions A, 47(6), 3165-3177.

Nomani, J., Pramanik, A., Hilditch, T., \& Littlefair, G. (2017). Stagnation Zone during the Turning of Duplex SAF 2205 Stainless Steels Alloy. Materials and Manufacturing Processes(just-accepted).

O'Sullivan, D., \& Cotterell, M. (2002). Machinability of austenitic stainless steel SS303. Journal of Materials Processing Technology, 124(1), 153-159.

Park, S. (1996). Robust design and analysis for quality engineering, chapman\& hall: London.

Paro, J., Hänninen, H., \& Kauppinen, V. (2001). Tool wear and machinability of HIPed P/M and conventional cast duplex stainless steels. Wear, 249(3), 279-284.

Pramanik, A. (2014). Developments in the non-traditional machining of particle reinforced metal matrix composites. International Journal of Machine Tools and Manufacture, 86, 44-61.

Pramanik, A., \& Basak, A. (2016). Degradation of wire electrode during electrical discharge machining of metal matrix composites. Wear, 346, 124-131.

Pramanik, A., Basak, A., Dixit, A., \& Chattopadhyaya, S. (2018). Processing of duplex stainless steel by WEDM. Materials and Manufacturing Processes, 1-9.

Pramanik, A., Basak, A., Dong, Y., Sarker, P., Uddin, M., Littlefair, G., . . Chattopadhyaya, S. (2017). Joining of carbon fibre reinforced polymer (CFRP) composites and aluminium alloys-A review. Composites Part A: Applied Science and Manufacturing, 101, 1-29.

Pramanik, A., Basak, A., Islam, M. N., \& Littlefair, G. (2015). Electrical discharge machining of 6061 aluminium alloy. Transactions of nonferrous metals society of China, 25(9), 2866-2874.

Pramanik, A., Islam, M. N., Boswell, B., Basak, A. K., Dong, Y., \& Littlefair, G. (2016). Accuracy and finish during wire electric discharge machining of metal matrix composites for different reinforcement size and machining conditions. Proceedings of the Institution of Mechanical Engineers, Part B: Journal of Engineering Manufacture, 0954405416662079. 
Pramanik, A., \& Littlefair, G. (2016). Wire EDM Mechanism of MMCs with the Variation of Reinforced Particle Size. Materials and Manufacturing Processes, 31(13), 1700-1708.

Pramanik, A., Littlefair, G., \& Basak, A. (2015). Weldability of duplex stainless steel. Materials and Manufacturing Processes, 30(9), 1053-1068.

Singh, H., \& Singh, A. (2012). Effect of pulse on/pulse off time on machining of AISI D3 die steel using copper and brass electrode in EDM. Int. J. of Engg. and Science, 1(9), 19-22.

Singh, H., \& Singh, E. (2013). Effect of Pulse on/Pulse Off on Machining of Steel Using Cryogenic Treated Copper Electrode.

Taguchi, G. (1987). System of Experimental Design. Engineering Methods to Optimize Quality and Minimize Cost (Vol. 2). White Plains, NY, USA: UNIPUB/Kraus Int. Pub.

Voronenko, B. (1997). Austenitic-ferritic stainless steels: A state-of-the-art review. Metal Science and Heat Treatment, 39(10), 428-437.

Williams, J. E., \& Rollason, E. C. (1970). Metallurgical and Practical Machining Parameters Affecting Built-up Edge Formation in Metal Cutting. Journal of the Institute of Metals, 98, 144-159. 